\title{
Quasiparticle band structures and lifetimes in noble metals using Gaussian orbital basis sets
}

\author{
Zhijun Yi, ${ }^{1}$ Yuchen Ma, ${ }^{1, *}$ Michael Rohlfing, ${ }^{1}$ V. M. Silkin, ${ }^{2,3,4,5}$ and E. V. Chulkov ${ }^{2,3,4}$ \\ ${ }^{1}$ Fachbereich Physik, Universität Osnabrück, Barbarastraße 7, 49069 Osnabrück, Germany \\ ${ }^{2}$ Donostia International Physics Center (DIPC), Paseo Manuel de Lardizabal 4, 20018 San Sebastián/Donostia, Basque Country, Spain \\ ${ }^{3}$ Departamento de Física de Materiales, Facultad de Ciencias Químicas, Universidad del Pais Vasco/Euskal Herriko Unibertsitatea, \\ Apdo. 1072, 20080 San Sebastián/Donostia, Basque Country, Spain \\ ${ }^{4}$ Centro de Física de Materiales CFM-Materials Physics Center MPC, Centro Mixto CSIC-UPV/EHU, Edificio Korta, \\ Avenida de Tolosa 72, 20018 San Sebastián, Spain \\ ${ }^{5}$ IKERBASQUE, Basque Foundation for Science, 48011 Bilbao, Spain \\ (Received 27 January 2010; revised manuscript received 5 March 2010; published 26 March 2010)
}

\begin{abstract}
We present the calculations of quasiparticle bandstructures and lifetimes for noble metals $\mathrm{Cu}$ and $\mathrm{Ag}$ within the $G W$ approximation based on localized Gaussian orbital basis sets. For $\mathrm{Cu}$, both the calculated positions of the $d$ bands and the width of the $d$ bands are within $0.1 \mathrm{eV}$ compared to the experimental results. For Ag, partial core correction should be included in the pseudopotential to get reliable positions of the $d$ bands. The calculated lifetimes agree with the experiment in the energy region away from the Fermi level, but deviate from the experimental results near the Fermi level where short-range interactions which $G W$ approach fails to describe play an important role. For a better description of the lifetime near the Fermi level, terms beyond the $G W$ approximation in the many-body perturbation theory need to be considered.
\end{abstract}

DOI: 10.1103/PhysRevB.81.125125

PACS number(s): 71.15.Ap, 71.20.-b

\section{INTRODUCTION}

In the last decade the electronic band structures and electron scattering in noble metals have been studied using different experimental techniques ${ }^{1-3}$ and a variety of theoretical methods. ${ }^{4-10}$ The $G W$ approximation derived by Hedin ${ }^{11}$ in 1965 has become the state-of-the-art approach to study the quasiparticle band structures and lifetimes of a large number of systems ${ }^{4-10,12-14}$ going beyond the well-known densityfunctional theory (DFT).

It is well-known that the usual local density approximation (LDA) (Ref. 15) underestimates the band gaps for the semiconductors, which can be improved by using the socalled scissors operator (i.e., by rigidly shifting the conduction bands to higher energy). However, for the noble metals the quasiparticle corrections are both $k$ point and band dependent, ${ }^{9}$ so a scissors operator is useless. Another important drawback of the traditional DFT is that the exchangecorrelation part is real and hence lifetime is infinite, whereas the lifetime of excited electrons and holes in metals is known to be finite.

A significant numerical problem when performing $G W$ calculations for noble metals is given by the issue of basis set expansion. The presence of strongly localized $d$ bands and the inclusion of semicore states for $\mathrm{Cu}$ and $\mathrm{Ag}$ make the $a b$ initio calculations based on a plane-wave expansion basis much heavier than those for semiconductors. For the case of $\mathrm{Cu}$, plane waves with cut-off energy of at least $160 \mathrm{Ry}$ (about 2700 plane waves) are needed for convergence. ${ }^{9} G W$ calculations need larger computational requirements than DFT, in particular for the calculations of surfaces and bulk solids with occupied $d$ orbitals. ${ }^{16}$ It turned out that a Gaussian orbital basis set instead of using plane-wave Fourier representations of the relevant two-point functions is efficient for the evaluation of $G W$ self-energy corrections. ${ }^{16}$ Another common simplification, i.e., the plasmon-pole approximation of the frequency dependence of $\epsilon(\omega)$ should be avoided for the $G W$ calculation of $\mathrm{Cu},{ }^{9}$ which means that the dielectric ma- trix $\epsilon_{G G^{\prime}}^{-1}(q ; \omega)$ should be computed explicitly by performing the energy integral numerically. This imposes further heavy CPU load for the $G W$ calculation with plane waves.

Most previous lifetime calculations are based on the DFT band energies. ${ }^{4-7,18}$ Furthermore, the so-called "energy shell" approximation with renormalization factor equal to unity was employed to obtain the imaginary part of the quasiparticle energies. In fact, the renormalization factor for noble metals varies between 0.57 and $0.77 .^{8}$ The renormalization factor directly changes the quasiparticle energies, and hence, changes the lifetime. Because of the large discrepancies between DFT $d$-band energies and experiments, the calculated lifetimes based on the DFT energies are not consistent with the experiment. In order to compare the calculated lifetimes based on the DFT band energies with experiments, the DFT band energies have to be shifted rigidly (which, as mentioned above, is questionable in metals).

So far the quantitative understanding of excitation mechanisms and their relaxation channels is far from being complete. In many cases the $G W$ approach correctly reproduces the changes of experimental electron relaxation times with the excitation energy. ${ }^{14}$ However, large discrepancies between theoretical and experimental results occur for $d$ holes in $\mathrm{Cu}$ and $\mathrm{Ag}$. The lifetimes of holes at the top of $d$ bands as calculated in Refs. 6 and 8 appear to be almost twice longer than the experimentally derived lifetimes. In Ref. 19 it was argued that due to the shifted $d$ bands in the DFT calculation the available phase space for Auger recombination is underestimated yielding calculated lifetimes which are too long. However, Zhukov et al., ${ }^{8,20}$ conclude that the drawbacks of the LDA band structures do not lead to qualitative changes of the quasiparticle lifetimes. The quasiparticle lifetime calculations based on the exact band energies have been performed for $\mathrm{Cu}$ using plane-waves method. ${ }^{21}$ However, because of large requirements of the computation, only several lifetimes at high-symmetry points were calculated. In Ref. 8 the lifetimes were calculated using the corrected local den- 
sity approximation, but the $d$ band threshold energies $(1.7 \mathrm{eV}$ for $\mathrm{Cu}, 3.2 \mathrm{eV}$ for $\mathrm{Ag}$ ) reported in this paper are still close to the Fermi level, compared to the photoemission data about 2 $\mathrm{eV}$ for $\mathrm{Cu}$ and about $3.8 \mathrm{eV}$ for $\mathrm{Ag}$. So it is necessary to calculate the lifetimes based on the accurate quasiparticle band energies.

In this paper, we go beyond the "energy shell" approximation to calculate the lifetimes of electrons and holes for $\mathrm{Cu}$ and $\mathrm{Ag}$ based on the accurate $G W$ band structure energies, using the efficient localized Gaussian orbitals. First, we discuss the quasiparticle band structures for $\mathrm{Cu}$ and $\mathrm{Ag}$. Then we analyze the influence of the quasiparticle corrections to the lifetimes. Our calculation starts with DFT evaluation of the ground-state properties performed using normconserving pseudopotentials, then the calculated DFT band structure energies and wave functions are used within the $G W$ approximation ( $G W A$ ) framework for the calculation of the self-energy operator, whose real part yields the quasiparticle band energies and imaginary part yields the lifetimes.

One particular aim of our work is to provide an approach to both band structure energies and their lifetimes within one calculation within the $G W$ approximation for the self-energy. For computational purposes many calculations so far have focused on either the band structure (which can often be obtained within the plasmon-pole approximation of the $G W A$, leading to a real self-energy and thus infinite lifetime) or on the lifetime (which can be done efficiently by considering a limited number of bands in the calculation). Here we show that both quantities are in fact obtained when the complex nature of the dielectric screening and the self-energy are treated carefully.

The paper is organized as follows. In Sec. II, we discuss some details of the theory of the GWA and summarize the basic equations needed for the following discussions. In Sec. III, the quasiparticle band structures for $\mathrm{Cu}$ and $\mathrm{Ag}$ are discussed. In Sec. IV, we present the quasiparticle lifetimes for $\mathrm{Cu}$ and $\mathrm{Ag}$ based on the accurate quasiparticle energies. A short summary concludes the paper in Sec. V.

\section{THEORY OF $G W$ APPROXIMATION}

The starting point of the calculation of band structures and lifetimes is given by a DFT calculation (referring to the electronic ground state), in which the Kohn-Sham equation

$$
\left.\left\{-\frac{\hbar^{2}}{2 m} \nabla^{2}+V_{P S}(\mathbf{r})+V_{H}(\mathbf{r})+V_{X C}(\mathbf{r})\right)\right\} \Psi_{n k}(\mathbf{r})=E_{n k} \Psi_{n k}(\mathbf{r}),
$$

is solved. In here, $V_{P S}$ and $V_{H}$ are the ions and Hartree potential, respectively. Since the $d$ electrons have to be considered as valence electrons, the entire shell to which they belong must be included as valence states throughout the calculations. ${ }^{9,17}$ For Ag we employ a norm-conserving pseudopotentia1 constructed along Hamann's scheme, ${ }^{22}$ based on the ionic configuration $4 s^{2} 4 p^{6} 4 d^{10}$. For $\mathrm{Cu}$, on the other hand, the transferability of such a pseudopotential seems not guaranteed. Therefore we employ a multireference pseudopotential (MRPP) constructed along Reis' scheme, ${ }^{23}$ based on the configuration $3 s^{2} 3 p^{6} 3 d^{10} 4 s^{1}$. Gaussian orbitals are used to construct the LDA basis sets. We use 50 Gaussian orbitals of $s, p, d$ and $s^{*}$ type for $\mathrm{Cu}$ atom and 60 Gaussian orbitals of $s, p, d$, and $s^{*}$ type for $\mathrm{Ag}$ atom. The decay constants are $0.18,0.50,1.24,3.2$, and 8.5 in units of $a_{0}^{-2}$ for $\mathrm{Cu}$, and $0.16,0.50,1.40,3.00,6.5$, and 14.0 in units of $a_{0}^{-2}$ for $\mathrm{Ag}$, respectively.

Based on the DFT results, the final treatment of band structures and lifetimes is done within many-body perturbation theory (MBPT). ${ }^{24}$ Assuming that the electronic spectrum is still given by well-defined quasiparticles, excited holes and electrons are described by an equation

$$
\begin{aligned}
& \left\{-\frac{\hbar^{2}}{2 m} \nabla^{2}+V_{P S}(\mathbf{r})+V_{H}(\mathbf{r})\right\} \Psi_{n k}^{Q P}(\mathbf{r}) \\
& \quad+\int \Sigma\left(\mathbf{r}, \mathbf{r}^{\prime}, E_{n k}^{Q P}\right) \Phi_{n k}^{Q P}\left(\mathbf{r}^{\prime}\right) d^{3} r^{\prime}=E_{n k}^{Q P} \Psi_{n k}^{Q P}(\mathbf{r}) .
\end{aligned}
$$

Compared to the KS equation, the local, real, and Hermitian exchange-correlation part is replaced by a nonlocal, complex and energy dependent self-energy operator. The self-energy operator results from electronic many-body effects beyond the DFT part of the exchange correlations. In particular, it contains exchange and correlation effects (relevant for the band structure energies) as well as Coulomb-scattering effects (relevant for the resulting lifetimes). Consequently, the self-energy operator leads to complex quasiparticle energies, i.e., the real part denotes the band structure energy while the imaginary part yields the inverse of the lifetime.

We calculate the self-energy within the $G W$ approximation of many-body perturbation theory by retaining the first term in the series expansion of self-energy operator in terms of the screened Coulomb interaction $W$

$$
\Sigma\left(\mathbf{r}, \mathbf{r}^{\prime}, E\right)=\frac{i}{2 \pi} \int e^{i \omega 0^{+}} G\left(\mathbf{r}, \mathbf{r}^{\prime}, E-\omega\right) W\left(\mathbf{r}, \mathbf{r}^{\prime}, \omega\right) d \omega .
$$

Equation (2) has to be solved self-consistently. It turns out that for many systems the DFT wave functions agree remarkably well with the final $G W$ wave functions. ${ }^{25}$

$$
\Psi_{n k}^{Q P}(\mathbf{r}) \simeq \Psi_{n k}^{D F T}(\mathbf{r})
$$

Therefore, the self-energy operator can be constructed using the results of the LDA calculation (wave functions, energy spectrum, and electronic density) and an iterative treatment of Eq. (2) is not necessary. The quasiparticle energies are then simply given by

$$
E_{n k}^{Q P}=E_{n k}^{D F T}+\operatorname{Re}\left[\Sigma_{n k}\left(E_{n k}^{Q P}\right)\right]-V_{X C}^{n k},
$$

where $\quad \Sigma_{n k}\left(E_{n k}^{Q P}\right)=\left\langle n k\left|\Sigma_{n k}\left(\mathbf{r}, \mathbf{r}^{\prime}, E_{n k}^{Q P}\right)\right| n k\right\rangle \quad$ and $\quad V_{X C}^{n k}$ $=\left\langle n k\left|V_{X C}(\mathbf{r})\right| n k\right\rangle$.

Simultaneously the imaginary part of $\Sigma(E)$ can be considered, which yields the Coulomb-scattering lifetimes. The imaginary part of the self-energy corresponds to the quasiparticle lifetimes, and the probability of the scattering decay is given by

$$
\tau_{n k}^{-1}=2\left|\operatorname{Im}\left[\Sigma_{n k}\left(E_{n k}^{Q P}\right)\right]\right| .
$$

The numerical evaluation of the $G W$ self-energy operator for the present case of metals, aiming at its real part and its 
imaginary part on equal footing, is more demanding than the common techniques for $G W$ calculations which focus on the band structure alone, and is also more demanding than the evaluation of lifetimes alone.

One key quantity is given by the time-ordered polarizability of the system, $P=-i G G$, which we calculate from DFTLDA quantities. For a periodic system it can be represented by basis functions $\chi_{\beta}(\mathbf{q}, \mathbf{r})$, leading to a matrix representation

$$
\begin{aligned}
P_{\beta \beta^{\prime}}(\mathbf{q}, \omega)= & 2 \frac{1}{V} \sum_{\mathbf{k}} \sum_{m} \sum_{n} M_{\beta}^{m n}(\mathbf{k}, \mathbf{q})\left[M_{\beta^{\prime}}^{m n}(\mathbf{k}, \mathbf{q})\right]^{*} \Theta\left(E_{F}\right. \\
& \left.-E_{m \mathbf{k}}\right) \Theta\left(E_{n, \mathbf{k}+\mathbf{q}}-E_{F}\right) \\
& \times\left[\frac{1}{E_{m \mathbf{k}}-E_{n, \mathbf{k}+\mathbf{q}}-\omega+i 0^{+}}\right. \\
& \left.+\frac{1}{E_{m \mathbf{k}}-E_{n, \mathbf{k}+\mathbf{q}}+\omega+i 0^{+}}\right],
\end{aligned}
$$

with spatial integrals $M_{\beta}^{m n}(\mathbf{k}, \mathbf{q})$ $=\int \psi_{m \mathbf{k}}^{*}(\mathbf{r}) \chi_{\beta}^{*}(\mathbf{q}, \mathbf{r}) \psi_{n, \mathbf{k}+\mathbf{q}}(\mathbf{r}) d^{3} r$. The summation is restricted to bands and $\mathbf{k}$ points such that $|m, \mathbf{k}\rangle$ is occupied and $\mid n, \mathbf{k}$ $+\mathbf{q}\rangle$ is empty. The direct evaluation of Eq. (7) can be tricky due to the careful balance between reciprocal-lattice points and frequencies, both of which have to be from a finite grid. In particular, the correct maintenance of charge neutrality (i.e., correct sum of the occupations of all states) is difficult to control. Furthermore, the replacement of the infinitesimally small imaginary part (from $i 0^{+}$) by broadening is difficult to control. Last but not least, the direct evaluation of Eq. (7) for all desired frequencies $\omega$ (usually several hundred) constitutes a computational bottleneck.

Instead, we first calculate the imaginary part of $P$ [using $\left.1 /\left(a+i 0^{+}\right)=1 / a-i \pi \delta(a)\right]$, i.e.,

$$
\begin{aligned}
\operatorname{Im} P_{\beta \beta^{\prime}}(\mathbf{q}, \omega)= & -2 \pi \frac{1}{V} \sum_{\mathbf{k}} \sum_{m} \sum_{n} M_{\beta}^{m n}(\mathbf{k}, \mathbf{q})\left[M_{\beta^{\prime}}^{m n}(\mathbf{k}, \mathbf{q})\right]^{*} \\
& \times\left|f_{m \mathbf{k}}-f_{n, \mathbf{k}+\mathbf{q}}\right| \delta\left[\omega-\left(E_{m \mathbf{k}}-E_{n, \mathbf{k}+\mathbf{q}}\right)\right] .
\end{aligned}
$$

In here, the occupation numbers $f_{m \mathbf{k}}$ and $f_{n, \mathbf{k}+\mathbf{q}}$ result from the respective band structure energy. The most important advantage of focusing on $\operatorname{Im}(P)$ first is that a given combination of $|m, \mathbf{k}\rangle$ and $|n, \mathbf{k}+\mathbf{q}\rangle$ contributes to a very small number of values for $\omega$, only, thus reducing the total numerical demand by an order of magnitude or more.

In practice, an occupation number $f$ is not taken from a sharp step at the Fermi level, but includes spectral broaden- ing by a Gaussian broadening parameter, which corresponds to the finite k-point grid employed in all calculations. Simply speaking, a state near the Fermi level can be expected to exhibit band structure dispersion in $\mathbf{k}$ (which is not explicitly considered due to the finite grid) and to cross the Fermi level somewhere in the vicinity of the grid point. It is thus not reasonable to attribute an occupation of just 0 (or 1 ) to a state slightly above (or below) the Fermi level, but the occupation function should be smeared out for states near the Fermi level. This procedure allows to mimic finer k-point sampling than the one which is really employed, thus reducing the dependence of the final results on the k-point grid. In addition, the $\delta$ function with respect to $\omega$ must be replaced by a finite-width spectral function, as well. The evaluation using a finite k-point grid yields sharp, discrete lines in the spectrum, which would be smeared out by continuous k-point integration. Again, the effect of using k-point integration instead of simple grid sampling can be mimicked by spectral broadening (smearing) of $\delta\left[\omega-\left(E_{m \mathbf{k}}-E_{n, \mathbf{k}+\mathbf{q}}\right)\right]$. The convergence of the spectral broadening has been tested carefully, $15 \mathrm{meV}$ yields converged results both for bandstructures and lifetimes, when the value of the spectral broadening is reduced from 75 to $15 \mathrm{meV}$.

After calculating the imaginary part of $P$ by carrying out the double summation of states $|m, \mathbf{k}\rangle$ and $|n, \mathbf{k}+\mathbf{q}\rangle$, its real part is simply obtained by a Kramers-Kronig transformation. All these operations are performed using a grid of 200 frequencies from zero to $100 \mathrm{eV}$ for $\mathrm{Cu}$, and a grid of 150 frequencies from zero to $100 \mathrm{eV}$ for $\mathrm{Ag}$. Negative frequencies need not be considered explicitly since $P(-\omega)=P(\omega)$.

Regardless of the above-mentioned issues concerning band structure summation, broadening etc., the polarizability must also be expanded in a real-space basis $\{\beta\}$ to represent its spatial properties. Here we employ a second ("auxiliary") basis of Gaussian orbitals (" $G W$ basis"). This $G W$ basis set must contain functions of higher angular momenta than the LDA basis set. ${ }^{16}$ We use 65 Gaussian orbitals of $s, p, d, s^{*}, f$, and $g$ type for $\mathrm{Cu}$ atom and 80 Gaussian orbitals of $s, p, d$, $s^{*}, f$, and $g$ type for $\mathrm{Ag}$ atom. A suitable choice of decay constants for these basis functions is given by $0.18,0.65$, and 2.65 in units of $a_{0}^{-2}$ for $\mathrm{Cu}$, and $0.18,0.65$, and 3.00 in units of $a_{0}^{-2}$ for $\mathrm{Ag}$, respectively.

After transforming the polarizability into the dynamically screened Coulomb interaction $W$, the self-energy operator is constructed. While its exchange part is quite simple to obtain, the correlation contribution to $\Sigma(E)$ is more difficult. Its expectation value for a given state is evaluated as

$$
\begin{aligned}
\left\langle m \mathbf{k}\left|\Sigma_{c}(E)\right| m^{\prime} \mathbf{k}\right\rangle & =\sum_{\mathbf{q}, n, \beta \beta^{\prime}} M_{\beta}^{\mathrm{mn}}(\mathbf{k}, \mathbf{q})\left(M_{\beta^{\prime}}^{\mathrm{m}^{\prime} \mathrm{n}}(\mathbf{k}, \mathbf{q})\right)^{*} \times \frac{i}{2 \pi} \int e^{-i \omega 0^{+}} \frac{W_{\beta \beta^{\prime}}(-\mathbf{q}, \omega)-v_{\beta \beta^{\prime}}(-\mathbf{q})}{E-\omega-E_{n, \mathbf{k}+\mathbf{q}}+i 0^{+} \operatorname{sign}\left(E_{n, \mathbf{k}+\mathbf{q}}-E_{F}\right)} d \omega \\
& =\sum_{\mathbf{q}, n} \frac{i}{2 \pi} \int e^{-i \omega 0^{+}} \frac{F_{m m^{\prime} n}(\mathbf{k}, \mathbf{q}, \omega)}{E-\omega-E_{n, \mathbf{k}+\mathbf{q}}+i 0^{+} \operatorname{sign}\left(E_{n, \mathbf{k}+\mathbf{q}}-E_{F}\right)} d \omega
\end{aligned}
$$

with $F$ containing the screening potential $W-v$ evaluated between states $m, m^{\prime}$, and $n$. Owing to its origin from $P(\omega)$ (see above), 
$F$ has a form (again within time-ordered Green-function theory) of ${ }^{16}$

$$
F_{m m^{\prime} n}(\mathbf{k}, \mathbf{q}, \omega)=\sum_{j} a_{j}\left(\frac{1}{\omega-\left(\omega_{j}-i 0^{+}\right)}-\frac{1}{\omega+\left(\omega_{j}-i 0^{+}\right)}\right)
$$

(omitting the dependence of $a_{j}$ and $\omega_{j}$ on $m, m^{\prime}, n, \mathbf{k}$, and $\mathbf{q}$ ) having real amplitudes $a_{j}$ and poles at frequencies $\pm\left(\omega_{j}\right.$ $-i 0^{+}$) with $\omega_{j}$ being real and positive. The self energy thus results as

$$
\left\langle m \mathbf{k}\left|\Sigma_{c}(E)\right| m^{\prime} \mathbf{k}\right\rangle=\sum_{\mathbf{q}, n} \sum_{j} a_{j} \times\left\{\begin{array}{ll}
\frac{1}{E+\left(\omega_{j}-i 0^{+}\right)-E_{n, \mathbf{k}+\mathbf{q}}} & \text { if }|n, \mathbf{k}+\mathbf{q}\rangle \text { is occupied } \\
-\frac{1}{E-\left(\omega_{j}-i 0^{+}\right)-E_{n, \mathbf{k}+\mathbf{q}}} & \text { if }|n, \mathbf{k}+\mathbf{q}\rangle \text { is empty }
\end{array} .\right.
$$

Consequently, the imaginary part of the self energy is given by

$$
\operatorname{Im}\left\langle m \mathbf{k}\left|\Sigma_{c}(E)\right| m^{\prime} \mathbf{k}\right\rangle=\sum_{\mathbf{q}, n} \sum_{j} a_{j} \times\left\{\begin{array}{c}
\pi \delta\left(E+\omega_{j}-E_{n, \mathbf{k}+\mathbf{q}}\right) \quad \text { if }|n, \mathbf{k}+\mathbf{q}\rangle \text { is occupied } \\
\pi \delta\left(E-\omega_{j}-E_{n, \mathbf{k}+\mathbf{q}}\right) \quad \text { if }|n, \mathbf{k}+\mathbf{q}\rangle \text { is empty }
\end{array},\right.
$$

which is immediately identified as

$$
\operatorname{Im}\left\langle m \mathbf{k}\left|\Sigma_{c}(E)\right| m^{\prime} \mathbf{k}\right\rangle=\sum_{\mathbf{q}} \sum_{\mathrm{n}}^{\prime} \operatorname{Im} F_{m m^{\prime} n}\left(\mathbf{k}, \mathbf{q},\left|E-E_{n, \mathbf{k}+\mathbf{q}}\right|\right),
$$

with the sum over $n$ restricted to such states $|n, \mathbf{k}+\mathbf{q}\rangle$ that are located between $E$ and the Fermi level. On the one hand, this expression reflects the physical mechanism, i.e., the imaginary part of the self-energy at energy $E$ is related to scattering events [contained in $\operatorname{Im}(W)$ and thus in $\operatorname{Im}(F)]$ involving final states between $E$ and $E_{F}$ (i.e., both quasielectrons and quasiholes are inelastically scattered toward the Fermi level). On the other hand, Eq. (12) provides an efficient way of calculating the self energy: we first evaluate its imaginary part (for frequencies $E$ on a real grid), from which the real part of $\Sigma$ is obtained via Kramers-Kronig relation afterwards. Again, the evaluation of the sum in Eq. (12), which includes or excludes states $n$ according to their energy relative to $E$ and $E_{F}$, is realized by employing spectral broadening of $E_{n, \mathbf{k}+\mathbf{q}}$, i.e., if $E_{n, \mathbf{k}+\mathbf{q}}$ is close to the Fermi level or close to $E$, it is included in the sum with fractional weight between 0 and 1 , mimicing the dispersion of $E_{n}$ in $\mathbf{k}+\mathbf{q}$.

\section{QUASIPARTICLE BAND STRUCTURES}

In this section, we discuss the band structures resulting from the quasiparticle theory outlined above.

\section{A. Copper}

Figure 1 displays the band structure of $\mathrm{Cu}$, as obtained within DFT (dashed curves) and within GWA (solid curves). In here, 50 Gaussian orbitals are used for representing the wave functions and 65 Gaussians for the polarizability and related quantities. Bandstructure energies for $\mathrm{Cu}$ at selected high-symmetry points are given in Table I, as well. For comparison, the table also contains data from a different quasi- particle calculation ${ }^{9}$ (employing a plane waves basis) and from experimental photoemission data. ${ }^{26}$

Exchange-correlation contributions to the self-energy arising from $3 s$ and $3 p$ core levels in copper are shown to be crucial, ${ }^{9}$ so we include the whole third atomic shell into the valence in the pseudopotential generation.

As can be seen from Fig. 1, the calculated $d$ band threshold within DFT amounts to 1.5 to $1.7 \mathrm{eV}$, respectively, compared to quasiparticle data of 2.0 to $2.5 \mathrm{eV}{ }^{27}$ All $d$ states are shifted to lower energy by $0.5-0.6 \mathrm{eV}$, leading to an excellent agreement with the experimental results for the positions of the $d$ bands, as can be seen from Table I. Our calculated results deviate from the experiments by less than $0.1 \mathrm{eV}$ except for the $L_{2}$, state. This indicates that $\mathrm{Cu}$ is very well described within the GWA. We also compare our calculations and the calculated data using plane waves reported in Ref. 9. For the widths of bands, our results employing Gaussian basis sets are close to the results of Ref. 9. A big difference between our result and the result of Ref. 9 occurs

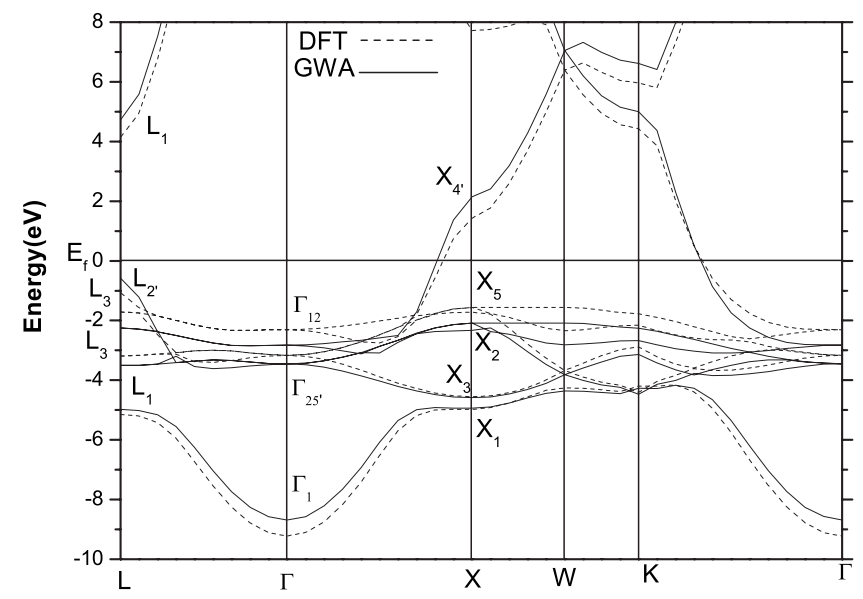

FIG. 1. Calculated band structure for $\mathrm{Cu}$ along high-symmetry lines, obtained within DFT (dashed lines) and within GWA (solid lines). 
TABLE I. Calculated bandwidths and band structure energies (in eV) for $\mathrm{Cu}$ at high-symmetry points. We show our DFT and GWA results in comparison. The values of the fifth column are GWA data from Ref. 9 using plane waves. The last column contains experimental data from Ref. 26.

\begin{tabular}{|c|c|c|c|c|c|}
\hline & State & DFT & $G W A$ & $G W A$ & Expt. \\
\hline & & This & Work & Ref. 9 & Ref. 26 \\
\hline \multirow[t]{3}{*}{ Positions of $d$ bands } & $\Gamma_{12}$ & -2.31 & -2.82 & -2.81 & -2.78 \\
\hline & $X_{5}$ & -1.56 & -2.09 & -2.04 & -2.01 \\
\hline & $L_{3}(2)$ & -1.71 & -2.24 & -2.24 & -2.25 \\
\hline \multirow{4}{*}{$\begin{array}{l}\text { Positions of } s / p \\
\text { Bands }\end{array}$} & $\Gamma_{1}$ & -9.22 & -8.68 & -9.24 & -8.60 \\
\hline & $X_{4^{\prime}}$ & 1.43 & 2.14 & & \\
\hline & $L_{1}$ & 4.14 & 4.73 & & \\
\hline & $L_{2}^{\prime}$ & -1.07 & -0.59 & -0.57 & -0.85 \\
\hline \multirow[t]{5}{*}{ Width of $d$ bands } & $\Gamma_{12}-\Gamma_{25^{\prime}}$ & 0.86 & 0.63 & 0.60 & 0.81 \\
\hline & $X_{5}-X_{3}$ & 2.99 & 2.50 & 2.49 & 2.79 \\
\hline & $X_{5}-X_{1}$ & 3.41 & 2.86 & 2.90 & 3.17 \\
\hline & $L_{3}^{(2)}-L_{3}^{(1)}$ & 1.47 & 1.27 & 1.26 & 1.37 \\
\hline & $L_{3}-L_{1}$ & 3.43 & 2.74 & 2.83 & 2.91 \\
\hline
\end{tabular}

at the $\Gamma_{1}$ point. The value of Ref. 9 is smaller than experiment by $0.64 \mathrm{eV}$, while our quasiparticle energy deviates from experiment only by $0.08 \mathrm{eV}$. All these results indicate that the localized Gaussian basis sets are efficient both for DFT and GWA calculations, especially for the GWA calculation of materials with occupied $d$ orbitals or other semicore states, which require a heavy CPU load with plane waves. For the case of $\mathrm{Cu}$, we only need 65 Gaussian orbitals for convergence. We note in passing that for a given state the QP shift does not simply result from the difference between $\Sigma$ and $V_{x c}$ alone, but also includes the recalibration of the Fermi level (which is shifted up by $0.27 \mathrm{eV}$ due to $\Sigma-V_{x c}$ ).

We also find that the values of the quasiparticle corrections (the differences between DFT energies and quasiparticle energies) change with the different bands and $k$ points, and at the $L$ point the shifts evenly change sign for different valence bands, with $\mathrm{QP}$ corrections ranging from $-0.53 \mathrm{eV}$ to $+0.52 \mathrm{eV}$. This indicates that the so-called scissors operator often used in LDA for semiconductor systems is not suitable for noble metals. These results are consistent with the results of Ref. 9. In short, the $G W$ self-energy corrections lead to excellent agreement with the experimental data.

\section{B. Ag}

Our calculated DFT band structures and the full GWA band structures for Ag are shown in Fig. 2. Some characteristic energy differences relating mostly to the occupied bands are given in Table II. This table also contains results from a different QP calculation ${ }^{10}$ and photoemission data ${ }^{28}$ for comparison.

Since the atomic structure of $\mathrm{Ag}$ is similar to the $\mathrm{Cu}$, we also include the $4 s$ and $4 p$ states to the valence for $\mathrm{Ag}$ in the pseudopotential generation. Though the semicore states are included, we find out that partial core correction (PCC) (Ref. 29) cannot be neglected for Ag. The DFT band energies of $\mathrm{Ag}$ are changed by $0.2 \mathrm{eV}$ with the inclusion of partial core correction. Our DFT band energy at $X_{5}$ point is $-2.68 \mathrm{eV}$, and close to the result of $-2.70 \mathrm{eV}$ from a full-potential calculation (within LMTO). ${ }^{8}$ Based on the DFT calculation, our computed $d$ band threshold at the $X_{5}$ point is about $-3.80 \mathrm{eV}$ within $G W A$, compared to photoemission data of $3.97 \mathrm{eV}$. Our quasiparticle results are in quite good agreement with the photoemission data.

Like in the case of $\mathrm{Cu}$, the Fermi level observes a value of $\Sigma^{G W}-V_{x c}$ of about $1.0 \mathrm{eV}$, while the top position of the $d$ bands observes $\Sigma^{G W}-V_{x c}=-0.2 \mathrm{eV}$. In total, this results in a downward of shift of about $1.2 \mathrm{eV}$ of the top of the $d$ bands, and leads to excellent agreement with available photoemission data, as can be seen from Table II. It can be seen from Fig. 2 that the $G W$ corrections do not act as a rigid shift of the whole occupied band structure with respect to the unoccupied part. This is similar to the situation in $\mathrm{Cu}$. Note, however, that the bottom of the $s p$ band (at $\left.\Gamma_{1}\right)$ is shifted downwards by $0.5 \mathrm{eV}$ for $\mathrm{Ag}$, which is different from the upwards

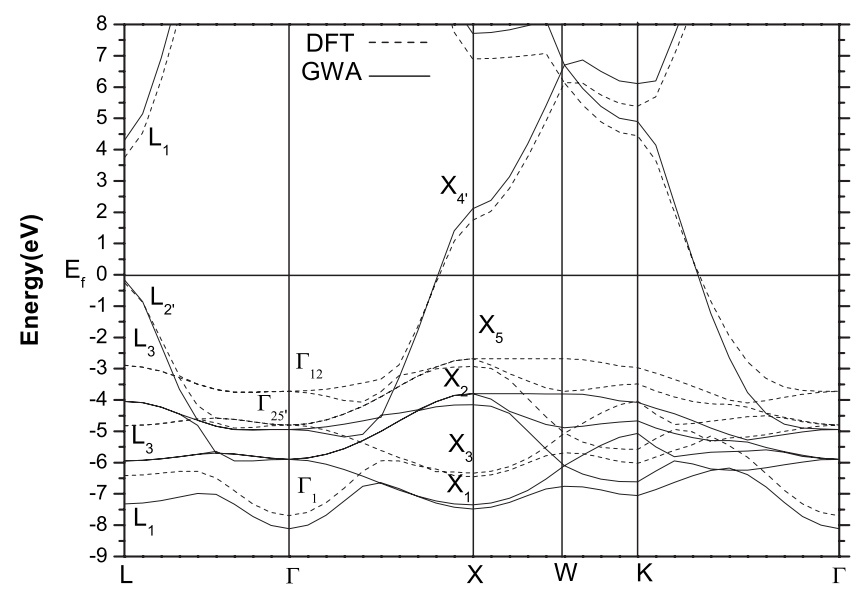

FIG. 2. Calculated band structure for Ag along high-symmetry lines, obtained within DFT (dashed lines) and within GWA (solid lines). 
TABLE II. Calculated bandwidths and band structure energies (in eV) for Ag at high-symmetry points. We show our DFT and GWA results in comparison. The values of the fifth column are GWA data from Ref. 10 using plane waves. The last column contains experimental data from Ref. 28.

\begin{tabular}{|c|c|c|c|c|c|}
\hline & State & DFT & $G W A$ & $G W A$ & Expt. \\
\hline & & This & Work & Ref. 10 & Ref. 28 \\
\hline \multirow[t]{3}{*}{ Positions of $d$ bands } & $\Gamma_{12}$ & -3.73 & -4.94 & -4.81 & -4.95 \\
\hline & $X_{5}$ & -2.68 & -3.80 & -3.72 & -3.97 \\
\hline & $L_{3}(2)$ & -2.90 & -4.05 & -3.94 & -4.15 \\
\hline \multirow[t]{6}{*}{ Width of $d$ bands } & $\Gamma_{12}-\Gamma_{25}$ & 1.08 & 0.97 & 0.94 & 1.11 \\
\hline & $X_{5}-X_{3}$ & 3.65 & 3.54 & 3.39 & 3.35 \\
\hline & $X_{5}-X_{1}$ & 3.77 & 3.61 & 3.51 & 3.40 \\
\hline & $X_{5}-X_{2}$ & 0.25 & 0.35 & 0.29 & 0.38 \\
\hline & $L_{3}^{(2)}-L_{3}^{(1)}$ & 1.92 & 1.89 & 1.85 & 1.99 \\
\hline & $L_{3}-L_{1}$ & 3.52 & 3.26 & 3.17 & 2.94 \\
\hline
\end{tabular}

shift in the case of $\mathrm{Cu}$. For the bandwidths of $d$ bands, our GWA results are closer to experiments than the DFT results are.

We also show calculated data using plane waves reported in Ref. 10 for comparison. In spite of the different methods of calculation, our calculated results are in good overall agreement with the results of Ref. 10 except for some minor differences. For example, our calculated quasiparticle energies of $d$ bands deviate from photoemission data by only about $0.1 \mathrm{eV}$, while the discrepancy is about $0.2 \mathrm{eV}$ in Ref. 10. For the case of $\mathrm{Ag}$, we get converged results using only 80 Gaussian orbitals.

\section{QUASIPARTICLE LIFETIMES}

Electron and hole dynamics in the bulk as well as at the various surfaces of a solid play a key role in a great number of physical and chemical phenomena. ${ }^{30,31} \mathrm{~A}$ path for the study of both electron and hole dynamics in the time domain was opened by the development of the time-resolved twophoton photoemission (TR-TPPE) technique. ${ }^{32,33}$ Recently first-principles calculations of the electron lifetimes in bulk noble metals have also been published in Refs. 4-8, 18, and 20. However, many problems related to the approximations employed still remain unresolved. The common problem is that these calculations are based on the DFT band energies. Another problem is that the renormalization factors are neglected in many $G W$ calculations such as Ref. 4, 6, and 18. In fact, the renormalization factor $\left[Z=\left(1-\Sigma^{\prime}\right)^{-1}\right]$ is quite far from the 1.0 used in the energy shell approximation. We got an average value of $Z=0.66$ for $\mathrm{Cu}$, and $Z=0.74$ for $\mathrm{Ag}$. This modifies the energy of the excitation, and hence, the lifetime.

\section{A. $\mathrm{Cu}$}

In Fig. 3 we compare our calculated lifetimes of electrons for $\mathrm{Cu}$ with the lifetimes of Ref. 7 and experimental data. ${ }^{35}$ The dashed lines in Fig. 3 are the lifetimes of Ref. 7 obtained within the so-called Fermi-liquid theory (FLT), the solid line represents the lifetimes of Ref. 7 calculated with plane waves, and the diamonds are the experimental data of Ref. 35 .

In spite of great differences in the methods of calculation, very good agreement is observed above $1.4 \mathrm{eV}$ between our data and the lifetimes based on the plane waves. Compared to the lifetimes obtained with plane waves, our calculated lifetimes are closer to the experimental results at about 1.1 $\mathrm{eV}$. Both our calculated electron lifetimes of hot electrons and the calculated lifetimes with plane waves are slightly larger than the results obtained within FLT. This is due to the influence of the $d$ electrons which provide an additional screening mechanism that is absent in the jellium model. Below $2 \mathrm{eV}$ the experimental data show a sudden increase. The origin of the disagreement between the calculated results and experimental data was discussed in Ref. 7, where it was explained that the increase is due to the interaction of the excited electron with its hole in the $d$ bands. As can be seen

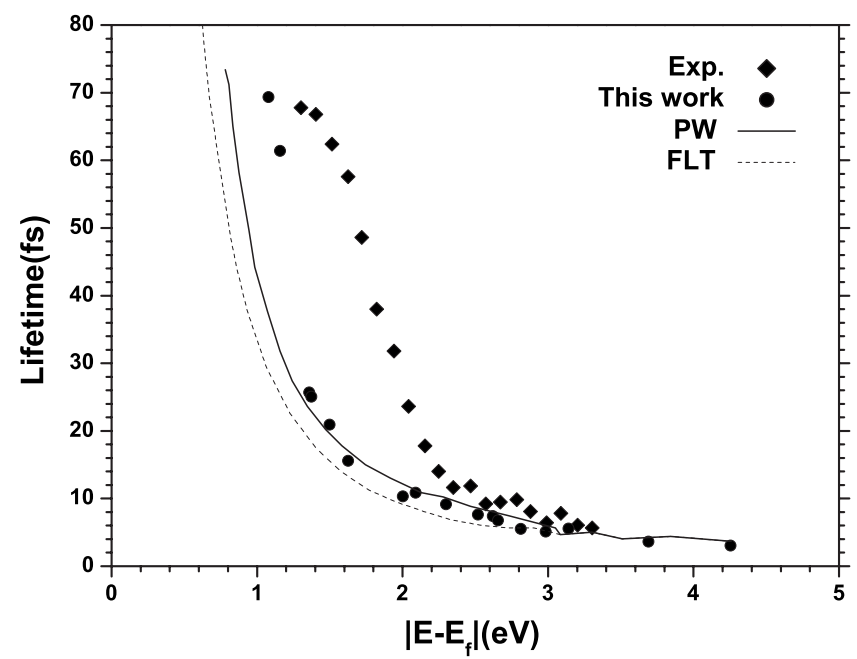

FIG. 3. Calculated lifetimes of hot electrons in $\mathrm{Cu}$ along highsymmetry lines (circles) in comparison with experimental data (diamonds). (Ref. 35) The solid and dashed lines denote theoretical results Ref. 7, obtained within GWA and within Fermi-liquid theory. 


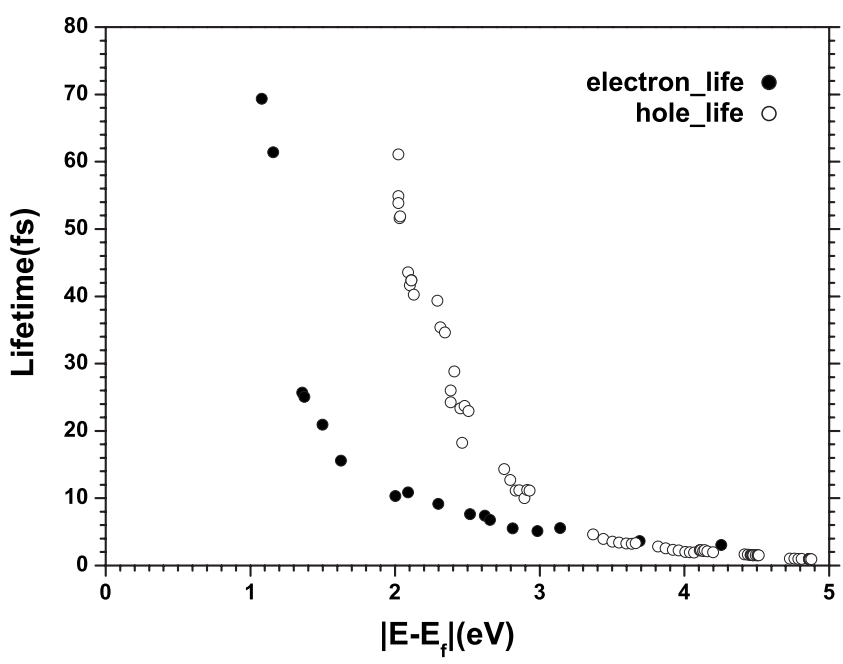

FIG. 4. Calculated lifetimes of $d$ holes in $\mathrm{Cu}$, at high-symmetry points. For comparison, the electron lifetimes are also presented (solid circles); open circles represent the hole lifetimes.

from Fig. 1, the threshold of $d$ bands is located about $2 \mathrm{eV}$ below Fermi level. The electrons at the top of $d$ bands can be excited by the photon energies widely used in 2PPE experiments, then the excited electrons interact with the holes in $d$ bands, and hence, the electron lifetimes increase at $2 \mathrm{eV}$ for $\mathrm{Cu}$. Ref. 34 compute the lifetimes for $\mathrm{Cu}$ with full inclusion of exchange and correlation (XC) effects based on the DFT band energies calculation. This approximation treats on the same footing XC effects between pairs of electrons within the Fermi sea (screening electrons) and between the excited hot electron and the Fermi sea. Though the inclusion of the high order terms of self-energy operator, the electron-hole interactions are still not included. They conclude that the overall effect of short-range $\mathrm{XC}$ is small and $G W \Gamma$ linewidths are close to their XC-free $G_{0} W_{0}$ counterparts. Our calculation is based on the accurate band energies and includes the semicore states. So the only one reason for the discrepancies between theoretical methods and experiments should be the interactions of the excited electrons with its holes in the $d$ bands, which are not included in the $G W$ calculation.

First-principle calculations based on the DFT predict an upper $d$-band edge that is located about $1.5 \mathrm{eV}$ below the
Fermi level, i.e., about $0.5 \mathrm{eV}$ higher than observed by photoemission experiments. ${ }^{26}$ This indicates that a rigid shift of the lifetime dependent energy will occur for the hole lifetime calculations based on the DFT band energies. The direct influence on the calculated lifetimes based on the DFT band energies is that the calculated values of $d$-hole lifetimes cannot be directly compared with experiment. Our calculation is based on the accurate band energies (i.e., the $d$ hole lifetimes at energies below the Fermi level by $2 \mathrm{eV}$ ), as shown in Fig. 4. It can also be seen from Fig. 4 that $d$ holes exhibit a longer lifetimes than $s p$ electrons with the same excitation energy, in agreement with experiment. As can be seen from Fig. 1, $s p$-like bands cross $d$ bands below the fermi level, and a small overlap occur between $s p$ states and $d$ states. The small overlap between $s p$ bands and $d$ bands is responsible for the increase in hole lifetimes at the top of $d$ bands. ${ }^{6}$

In order to find out the influence of quasiparticle corrections on the hole lifetimes, we compare our calculated $d$ hole lifetimes with the lifetimes of Refs. 8, 20, and 21 and the experiment, ${ }^{19}$ as shown in Table III. In Ref. 8, the linear muffin-tin orbitals (LMTO) method has been used. Ref. 20 employs full potential linear muffin-tin orbitals (FP LMTO) method. In the FP LMTO method the crystal space is separated into atomic spheres and interstitial region. Contrary to the LMTO ASA method, all the nonspherical components of the crystal potential in the interstitial region are taken into account. Inside the atomic spheres the LDA eigenfunctions are expanded into a set of muffin-tin orbitals and their energy derivatives. Inside the interstitial space the LDA eigenfunctions are expanded into a set of plane waves. In Ref. 21 the pseudopotential method with the plane-wave basis set has been used. The last column is the experimental data.

For the calculated $d$ hole lifetimes near the Fermi level, different methods yield different results, as can be seen from Table III. Our calculated $d$ hole lifetimes at $X_{5}$ and $X_{2}$ points within the on energy shell approximation are significantly larger than the results of experiment. Our method beyond on energy shell approximation leads to a decease of $d$ hole lifetimes at $X_{5}$ and $X_{2}$ points, but the results are still larger than the experimental data. In Ref. 21 the calculated $d$ hole lifetimes beyond on shell approximation at these points agree with the experimental data. In Ref. 20 the calculated $d$ hole lifetimes within the on shell approximation at $X_{5}$ and $X_{2}$ points slightly deviate from the experimental results. They

TABLE III. Lifetimes of $d$ holes in $\mathrm{Cu}$ (in fs).

\begin{tabular}{|c|c|c|c|c|c|c|c|c|c|}
\hline & Symmetry points & This work & This work & $\begin{array}{l}\text { ASA LMTO } \\
\text { Ref. } 8\end{array}$ & $\begin{array}{l}\text { FP LMTO } \\
\text { Ref. } 20\end{array}$ & $\begin{array}{l}\text { FP LMTO } \\
\text { Ref. } 20\end{array}$ & $\begin{array}{c}\text { PW } \\
\text { Ref. } 21\end{array}$ & $\begin{array}{c}\text { PW } \\
\text { Ref. } 21\end{array}$ & Expt. \\
\hline & & On shell & & & & On shell & & On shell & \\
\hline \multirow[t]{7}{*}{ Life time of $d$ states } & $X_{5}$ & 73.1 & 64.4 & 47.1 & 33.7 & 23.6 & 23.8 & 79.4 & $33 \pm 7$ \\
\hline & $X_{2}$ & 69.4 & 53.9 & 47.5 & 30.7 & 21.2 & 17.9 & 88.0 & 6.5 \\
\hline & $L_{3}(2)$ & 27.1 & 23.9 & 23.5 & 21.4 & 15.0 & & & \\
\hline & $\Gamma_{12}$ & 17.7 & 22.2 & 12.6 & 14.8 & 9.9 & 12.7 & 25.0 & 5.0 \\
\hline & $\Gamma_{25^{\prime}}$ & 4.0 & 6.2 & 3.2 & 6.4 & 4.2 & 6.7 & 6.8 & 3.7 \\
\hline & $L_{3}(1)$ & 4.1 & 6.0 & 3.0 & 6.4 & 4.2 & & & \\
\hline & $X_{3}$ & 1.0 & 1.7 & 0.6 & 2.3 & 1.5 & 2.3 & 1.1 & 2.8 \\
\hline
\end{tabular}




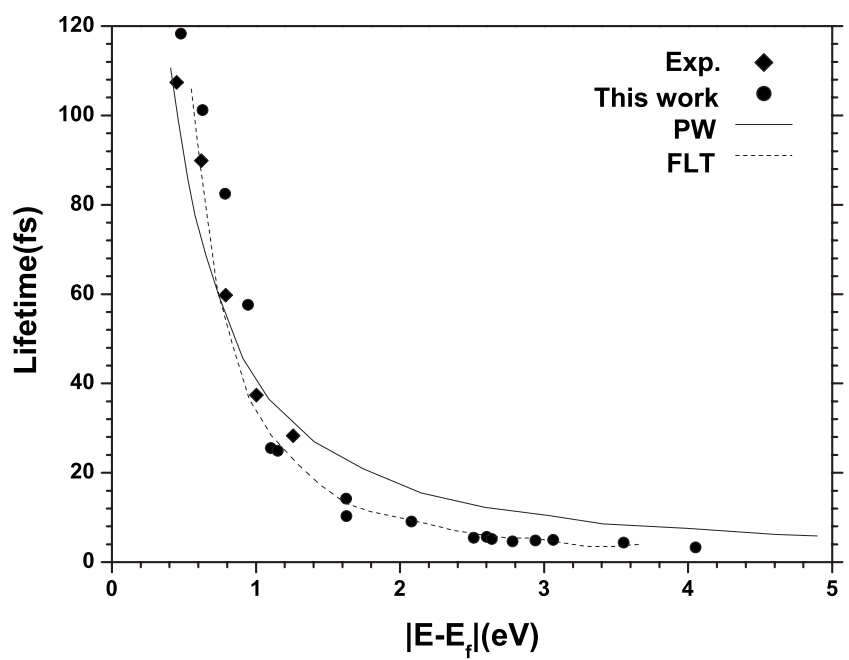

FIG. 5. Calculated lifetimes of hot electrons in Ag at highsymmetry points, solid circles show our results; solid diamonds represent experiments; (Ref. 35) solid line and dashed line denote the results of Ref. 7.

differ from our results and the results of Ref. 21 . The reason for these discrepancies is not clear. Ref. 19 reported that the calculated lifetimes below $3 \mathrm{eV}$ tend to approach those predicted by a FEG (free electron gas) model of the solid, the experiment show a distinct asymptotic behavior at the large binding energies. They attribute this discrepancy to the failure of the DFT to reproduce the actual band structure of $\mathrm{Cu}$. As can be seen from Table III, our calculated lifetime at $\Gamma_{25^{\prime}}$ (about $3.58 \mathrm{eV}$ below Fermi level) is larger than the experimental data. Both our calculated lifetime and the lifetime of Ref. 8 at $X_{3}$ (about $4.78 \mathrm{eV}$ below the Fermi level) are smaller than the experimental data, especially, the calculated lifetime of Ref. 8 deviate from experiment by 2.2 fs. The LDA eigenvalues have been corrected before entering the matrix elements of self-energy operator in Ref. 8. As can be seen from Table I and Fig. 1, the positions of $d$ bands are close to the experimental results. As can be seen from Table III, all theoretical lifetimes above $\Gamma_{25}$, deviate from experimental data. So the quasiparticle corrections to the DFT bandstructure energies are not essential reasons for the disagreements between theoretical lifetimes and experiments, and this is consistent with the conclusion of Ref. 20. In order to accurately describe lifetimes of holes for noble metals, $G W$ approximation should be improved.

\section{B. Ag}

Our calculated electron lifetimes for Ag are given in Fig. 5 (solid circles). We compare our results with the results of Ref. 7 based on the plane-wave bases sets, FLT results and experimental data. ${ }^{35}$

Our calculated lifetimes of electrons in Ag are well agreement with the results from literature. As can be seen from Fig. 5, our results are closer to the results obtained within FLT than the theoretical lifetimes with plane waves. As can be seen from Fig. 2, the $d$ band threshold locates about 3.80 $\mathrm{eV}$ below the Fermi level. This indicates that for all excited

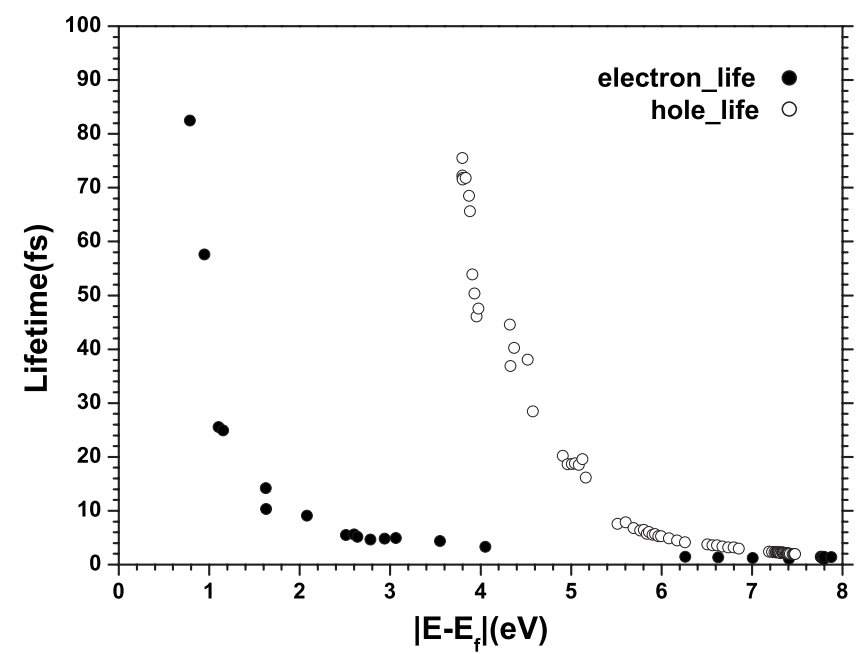

FIG. 6. Calculated lifetimes of $d$ holes in Ag, at high-symmetry points. Open circles represent the lifetime of holes; solid circles depict the lifetime of electrons.

electrons below $3.80 \mathrm{eV}$ the relaxation of the hot electrons cannot excite the electrons in the $d$ bands, and lead to FLT behavior of $\mathrm{Ag}$. Contrary to the case of $\mathrm{Cu}$, the experimental data for Ag do not show a sudden increase at small energies, due to the fact that the $d$ band threshold is about $3.80 \mathrm{eV}$ below the Fermi level, as shown in Fig. 2, and no $d$ electrons are excited for the photon energies widely used in 2PPE experiments. This indicates that the electron lifetimes of $\mathrm{Ag}$ are mainly attributed to the electron-electron interactions.

It can be seen from Fig. 6 that the hole lifetimes are larger than the electron lifetimes, which is in agreement with experiments, ${ }^{36}$ and this is similar to the case of $\mathrm{Cu}$. The band structure in Fig. 2 presents a small overlap between $d$ and $s p$ states at the top of $d$ bands (3.8 eV below the Fermi level) and large overlaps between $d$ bands at deeper energy below Fermi level. The small overlap yields a dramatic increase of the hole lifetimes, especially, at the top of $d$ bands. As the $d$ hole energy decreases, large overlap between $d$ bands yields a rapid decrease in hole lifetimes.

Unlike to the case of $\mathrm{Cu}$, our calculated $d$ hole lifetimes within on shell approximation are slightly smaller than the results beyond on shell approximation (Table IV). At $X_{5}$ point our calculated lifetime beyond on shell approximation is about $68 \mathrm{fs}$, while the experimental ${ }^{36}$ value is $22 \mathrm{fs}$. Large discrepancy appears at $X_{2}$ point. For the states at $X_{1}$, a good agreement is observed between our calculated lifetime beyond on shell approximation and lifetime measured by ex-

TABLE IV. Lifetimes of $d$ holes for $\mathrm{Ag}$ (in fs)

\begin{tabular}{|c|c|c|c|}
\hline \multirow[b]{2}{*}{ Symmetry points } & This work & This work & Expt. \\
\hline & \multicolumn{3}{|l|}{ On shell } \\
\hline$X_{5}$ & 53.7 & 68.8 & 22 \\
\hline$X_{2}$ & 65.4 & 74.2 & 12 \\
\hline$X_{3}$ & 1.6 & 2.2 & \\
\hline$X_{1}$ & 1.6 & 2.0 & 2.0 \\
\hline
\end{tabular}


periment. In spite of the large quasiparticle corrections of the $d$ bands for $\mathrm{Ag}(1.2 \mathrm{eV})$, our calculated hole lifetimes for $\mathrm{Ag}$ still deviate from experiment, and this is similar to the case of $\mathrm{Cu}$.

It is well-known that the $G W$ approximation well accounts for the long-range screening. The short-range interactions such as electron-hole and hole-hole interactions near Fermi level may be crucial for the hole lifetimes in the case of noble metals and electron lifetimes in the case of $\mathrm{Cu}$. With increasing distance to Fermi level, the long-range interactions dominate the scattering mechanism and thus lead to well agreement with the experiments.

\section{SUMMARY}

In conclusion, we have performed the first $a b$ initio calculations of quasiparticle band structures and lifetimes for noble metals $\mathrm{Cu}$ and $\mathrm{Ag}$ using efficient Gaussian orbital basis set. With the use of localized Gaussian orbitals, only less than 80 Gaussian orbitals both for the LDA basis sets and the GWA basis sets are needed, instead of thousands of plane waves. The traditional LDA calculations for $\mathrm{Cu}$ and $\mathrm{Ag}$ usually place $d$ bands too close to the Fermi level, especially for the Ag. The well-known discrepancies between experiments and DFT band structures are almost completely corrected by $G W$ calculations. It turns out that Hedin's $G W$ approximation is successful for the description of band structures of noble metals.

Based on the accurate band structures, the lifetimes of $\mathrm{Cu}$ and Ag have been calculated. Comparing with experimental data, the quasiparticle corrections do not lead to qualitative improvements of the quasiparticle lifetimes. It is well-known that the $G W$ approach well accounts for the long range screening, while short-range interactions which are more important for noble metals are not well-described. Therefore, it is probable that the improvement of the calculated lifetimes within $G W$ approximation for $d$ materials can be achieved by inclusion of higher terms of the many-body perturbation, i.e., the short-range screening such as electron-hole and hole-hole interactions should be included when we evaluate the selfenergy operator.

\section{ACKNOWLEDGMENTS}

This work has been supported by the Niedersachsen Ph. D. program "Synthesis and Characterization of Surfaces and Interfaces assembled from Clusters and Molecules" and by the Deutsche Forschungsgemeinschaft (Bonn, Germany) by Grant No. Ro 1318/4-3. *yuma@uos.de

${ }^{1}$ V. N. Strocov et al., Phys. Rev. Lett. 81, 4943 (1998).

${ }^{2}$ R. Matzdorf, A. Gerlach, F. Theilmann, G. Meister, and A. Goldmann Appl. Phys. B: Lasers Opt. 68, 393 (1999).

${ }^{3}$ H. Petek, H. Nagano, and S. Ogawa, Appl. Phys. B: Lasers Opt. 68, 369 (1999).

${ }^{4}$ I. Campillo, J. M. Pitarke, A. Rubio, E. Zarate, and P. M. Echenique, Phys. Rev. Lett. 83, 2230 (1999).

${ }^{5}$ W.-D. Schöne, R. Keyling, M. Bandic, and W. Ekardt, Phys. Rev. B 60, 8616 (1999).

${ }^{6}$ I. Campillo, A. Rubio, J. M. Pitarke, A. Goldmann, and P. M. Echenique, Phys. Rev. Lett. 85, 3241 (2000).

${ }^{7}$ R. Keyling, W. D. Schöne, and W. Ekardt, Phys. Rev. B 61, 1670 (2000).

${ }^{8}$ V. P. Zhukov, F. Aryasetiawan, E. V. Chulkov, I. G. de Gurtubay, and P. M. Echenique, Phys. Rev. B 64, 195122 (2001).

${ }^{9}$ A. Marini, G. Onida, and R. Del Sole, Phys. Rev. Lett. 88, 016403 (2001).

${ }^{10}$ A. Marini, R. Del Sole, and G. Onida, Phys. Rev. B 66, 115101 (2002).

${ }^{11}$ L. Hedin, Phys. Rev. 139, A796 (1965).

${ }^{12}$ V. P. Zhukov, F. Aryasetiawan, E. V. Chulkov, and P. M. Echenique, Phys. Rev. B 65, 115116 (2002).

${ }^{13}$ M. R. Bacelar, W.-D. Schöne, R. Keyling, and W. Ekardt, Phys. Rev. B 66, 153101 (2002).

${ }^{14}$ E. V. Chulkov, A. G. Borisov, J. P. Gauyacq, D. Sánchez-Portal, V. M. Silkin, V. P. Zhukov, and P. M. Echenique, Chem. Rev. (Washington, D.C.) 106, 4160 (2006).

${ }^{15}$ D. M. Ceperley and B. J. Alder, Phys. Rev. Lett. 45, 566 (1980);
J. P. Perdew and A. Zunger, Phys. Rev. B 23, 5048 (1981).

${ }^{16}$ M. Rohlfing, P. Krüger, and Johannes Pollmann, Phys. Rev. B 52, 1905 (1995).

${ }^{17}$ M. Rohlfing, P. Krüger, and J. Pollmann, Phys. Rev. Lett. 75, 3489 (1995).

${ }^{18}$ I. Campillo, V. M. Silkin, J. M. Pitarke, E. V. Chulkov, A. Rubio, and P. M. Echenique, Phys. Rev. B 61, 13484 (2000).

${ }^{19}$ A. Gerlach, K. Berge, A. Goldmann, I. Campillo, A. Rubio, J. M. Pitarke, and P. M. Echenique, Phys. Rev. B 64, 085423 (2001).

${ }^{20}$ V. P. Zhukov, E. V. Chulkov, and P. M. Echenique, Phys. Rev. B 68, 045102 (2003).

${ }^{21}$ A. Marini, R. Del Sole, A. Rubio, and G. Onida, Phys. Rev. B 66, 161104(R) (2002).

${ }^{22}$ D. R. Hamann, M. Schlüter, and C. Chiang, Phys. Rev. Lett. 43, 1494 (1979).

${ }^{23}$ C. L. Reis, J. M. Pacheco, and J. L. Martins, Phys. Rev. B 68 , 155111 (2003).

${ }^{24}$ L. Luttinger and J. Ward, Phys. Rev. 118, 1417 (1960).

${ }^{25}$ M. S. Hybertsen and S. G. Louie, Phys. Rev. B 34, 5390 (1986).

${ }^{26}$ S. Ogawa, H. Nagano, and H. Petek, Phys. Rev. B 55, 10869 (1997).

${ }^{27}$ R. Courths and S. Hüfner, Phys. Rep. 112, 53 (1984).

${ }^{28}$ G. Fuster, J. M. Tyler, N. E. Brener, J. Callaway, and D. Bagayoko, Phys. Rev. B 42, 7322 (1990).

${ }^{29}$ S. G. Louie, S. Froyen, and M. L. Cohen, Phys. Rev. B 26, 1738 (1982).

${ }^{30}$ H. Petek and S. Ogawa, Prog. Surf. Sci. 56, 239 (1997).

${ }^{31}$ Laser Spectroscopy and Photochemistry on Metal Surfaces, edited by H. L. Dai and W. Ho (World Scientific, Singapore, 
1995).

${ }^{32}$ J. Bokor, Science 246, 1130 (1989).

${ }^{33}$ R. Haight, Surf. Sci. Rep. 21, 275 (1995).

${ }^{34}$ I. G. Gurtubay, J. M. Pitarke, and P. M. Echenique, Phys. Rev. B
69, 245106 (2004).

${ }^{35}$ M. Wolf and M. Aeschlimann, Phys. Bl. 54, 145 (1998).

${ }^{36}$ A. Gerlach, K. Berge, T. Michalke, A. Goldmann, R. Müller, and C. Janowitz, Surf. Sci. 497, 311 (2002). 